\title{
COMERCIO Y CRECIMIENTO EN LA ECONOMÍA ESPAÑOLA, 1870-1995
}

\author{
EVA PARDOS*
}

Universidad de Zaragoza

\section{RESUMEN}

Este artículo presenta un análisis de las conexiones entre sector exterior y crecimiento en la economía española, a través del análisis de causalidad tipo Granger entre exportaciones y renta e importaciones y renta para tres períodos: 1870-1935, 1940-1959 y 1964-1995. Los resultados confirman una influencia positiva mutua, que actúa desde las exportaciones hacia el crecimiento a partir de los años cuarenta, y sólo desde los sesenta en el caso de las importaciones. La interpretación de ausencia de causalidad del comercio a la renta entre 1870 y 1935 se basa fundamentalmente en el tipo de composición del comercio exterior español en la época.

\section{ABSTRACT}

This paper presents an analysis of the relationship between foreign sector and growth in the Spanish economy, through Granger causality tests for exports and income, and imports and income, along three periods: 1870-1935, 1940-1959 and 1964-1995. The results confirm a positive mutual influence, which works from exports to growth from the 1940s onwards, and begins only in the $1960 \mathrm{~s}$ in the case of imports and growth. Our interpretation for the absence of causality from trade to growth between 1870 and 1935 stems mainly from the composition of Spanish trade at the time.

\section{INTRODUCCIÓN}

La teoría del comercio internacional se aleja en la actualidad de interpretaciones extremas del comercio como motor del crecimiento o como causante de un atraso persistente. Ni siquiera es evidente que a un nivel

* La autora desea agradecer las observaciones de dos evaluadores anónimos y las sugerencias de los participantes en el seminario Nuevas Interpretaciones en la Historia Económica de España, así como de Marcela Sabaté y de Dolores Gadea a versiones anteriores de este artículo. 
superior de desarrollo económico le corresponda necesariamente un mayor grado de apertura comercial. La responsabilidad del comercio en el crecimiento se ha ido rebajando a la de ser auxiliar en el proceso, o la de mero lubricante, en lugar de combustible, en consonancia con las recientes teorías de crecimiento endógeno que otorgan mayor relevancia a las innovaciones tecnológicas o al capital humano. Incluso la noción del crecimiento impulsado por exportaciones se acepta más como una excepción que como una estrategia generalizable a tódos los países en desarrollo. No obstante, y aun rebajado de énfasis, el debate continúa. En los últimos años han surgido abundantes aplicaciones que intentan contrastar las conexiones entre comercio y crecimiento, en unos casos, y entre liberalización comercial y crecimiento en otros, aunque con escasas aportaciones de tipo histórico ${ }^{1}$.

La contribución de este trabajo consiste en aplicar el análisis de causalidad entre crecimiento económico y comercio a la economía española en el largo plazo, caso apenas tratado en este tipo de investigaciones, y sólo para las décadas más recientes, a pesar de tratarse de relaciones relevantes para la discusión historiográfica sobre el atraso relativo español y las opciones disponibles para superarlo. El trabajo se enmarca en una bibliografía más amplia, que ha investigado el papel del comercio internacional, de la orientación comercial y de las políticas comerciales de los diversos países en sus posibilidades de crecimiento económico, y que en los últimos años se ha situado dentro del debate entre la teoría neoclásica del crecimiento y las explicaciones de crecimiento endógeno ${ }^{2}$. Sin embargo, como recuerdan Greenaway y Sapsford, los interrogantes deberían plantearse sucesivamente: cómo se relacionan exportaciones y crecimiento, y cómo influye la liberalización comercial en las exportaciones y en el crecimiento ${ }^{3}$. Es necesario contestar inicialmente a la primera pregunta, y es lo que nos proponemos en el presente trabajo, aplicándola también a la conexión entre importaciones y renta. Más concretamente, se trata de contrastar la relación entre la evolución de la renta y los flujos de exportaciones y de importaciones desde finales del siglo xxx hasta la actualidad, a través de un análisis de causalidad bivariante tipo Granger para tres períodos distintos: 1870-1935, 1940-1959 y 1964-1995. La hipótesis de partida es que en

${ }^{1}$ Nurkse (1964), Singer (1950) y Prebisch (1950). Véase también Kravis (1970) y Lewis (1978). Dos panoramas recientes con énfasis en los resultados empíricos son Edwards (1993) y Rodrik (1995).

2 Véase Barro y Sala-i-Martin (1995) como exposición de las diferentes teorías sobre el crecimiento, y Edwards (1993) respecto a los trabajos empíricos sobre apertura y crecimiento.

${ }^{3}$ Greenaway y Sapsford (1994). 
el largo plazo el comercio ha tenido una influencia positiva sobre el crecimiento, en particular, que las exportaciones han ayudado al crecimiento de la renta, mientras que es más probable que este último haya impulsado el crecimiento de las importaciones. No obstante, pueden esperarse diferentes comportamientos en las tres etapas contempladas, fundamentalmente en respuesta a la progresiva modernización de nuestro sector exterior desde los años sesenta y a los cambios en la restricción exterior al crecimiento español a lo largo del tiempo.

En el primer apartado se hace referencia a los análisis teóricos y empíricos sobre la relación entre comercio y crecimiento, y a su discusión en la historiografía española. El segundo apartado muestra las relaciones de causalidad encontradas en la aplicación (cuya presentación detallada se relega al Apéndice, p. 166). Estos resultados se comentan a modo de conclusión en el apartado final.

\section{COMERCIO Y CRECIMIENTO}

Las relaciones teóricas entre comercio y renta permiten una variedad de posibilidades que se resumen a continuación. Tradicionalmente la hipótesis de crecimiento impulsado por las exportaciones se ha apoyado con los siguientes argumentos: 1) a través de mejoras de eficiencia por incremento en la competencia; 2) relajando la restricción de divisas; 3) aumentando la calidad de los productos por contacto con los consumidores del resto del mundo; 4) mediante el incremento de empleo y salarios reales si la economía contaba con exceso de mano de obra; y 5) a través de una mayor productividad por la explotación de las economías de escala en la producción (cuando se incorpora la competencia imperfecta) y por la mayor difusión de conocimiento técnico, que permite incrementar la tasa de formación de capital y el cambio tecnológico - hipótesis ligadas a las teorías de crecimiento endógeno ${ }^{4}$-. También se han supuesto influencias en sentido contrario. La hipótesis de una causalidad de la renta hacia las exportaciones se basa en diversas relaciones posibles: una mayor productividad que reduzca los costes unitarios aumentará las exportaciones; por otra parte, si la producción interna aumenta más rápidamente que la demanda, las exportaciones son la vía de salida para dicha producción

${ }^{4}$ Véase Bhagwati y Srinivasan (1979), Krueger (1980), McKinnon (1964), Helpman y Krugman (1985), y Grossman y Helpman (1991). 
(modelos de crecimiento impulsado por la demanda) ${ }^{5}$. Incluso se han propuesto relaciones con signo negativo, por sesgos anti-comercio en el crecimiento o caídas del output por incrementos de exportaciones en economias con fuertes distorsiones.

Podría suceder que no hubiera relación de causalidad entre ambas variables, y que su crecimiento simultáneo fuera meramente resultado del proceso de desarrollo y cambio estructural. Sin embargo, sin negar estas influencias, el vínculo causal entre ambas variables parece probable dados los argumentos teóricos recién expuestos. Por último, también puede aparecer causalidad bidireccional entre importaciones y renta. Aunque tradicionalmente se considera a las importaciones como función de la renta nacional, pueden ser un determinante importante del crecimiento si suministran materias primas clave y aportan tecnología incorporada. Ante esta variedad de argumentos teóricos, la naturaleza de las relaciones de causalidad entre crecimiento y comercio, presentes en una economía en un momento concreto, se convierte en un asunto empírico, tanto en lo referente a su dirección como a su signo, y no puede descartarse una causalidad en dos direcciones.

Las primeras contrastaciones de estas hipótesis tomaron la forma de comparaciones entre países mediante análisis de convergencia de corte transversal, en los que las variables de sector exterior se añadían a las dotaciones factoriales $y / o$ las variables de política económica, para identificar su signo y nivel de significación. Más adelante, las técnicas de datos de panel permitieron incluir distintos cortes temporales en las muestras. De los abundantes trabajos de este tipo, se concluye un apoyo débil a la relación entre apertura y crecimiento, dando lugar a una discusión sobre la robustez de los resultados ${ }^{6}$. En particular, un problema repetidamente señalado en las estimaciones era la ausencia de características individuales de los países que permitieran distinguir su comportamiento.

Para solucionarlo, se realizan estimaciones individuales de series temporales por países, que además facilitan la contrastación de direcciones de causalidad. Pero en los primeros estudios, los problemas de estacionariedad llevaban a utilizar tasas de crecimiento en lugar de niveles, con la consiguiente pérdida de información sobre el largo plazo. Ello se ha superado con las técnicas recientes de tratamiento de series temporales, que permiten aclarar la relación entre el crecimiento económico y sus deter-

'Bhagwati (1988).

' Véase Levine y Renelt (1992) o Ghali (1998) como ejemplos. 
minantes, siempre que se analicen las propiedades temporales de dichas series para aplicar la técnica econométrica y los tests adecuados. Los análisis de cointegración y test de causalidad de Granger se han extendido en los últimos años, permitiendo su aplicación a diversos tipos de economías y períodos, aunque con predominio de países en desarrollo durante las últimas décadas ${ }^{7}$. Los resultados no apoyan definitivamente ninguna de las hipótesis planteadas, aunque predominan las relaciones positivas $\mathrm{y}$, en varias ocasiones, se detecta causalidad en dos direcciones. Apenas unos pocos extienden el período de estudio hasta el siglo XIX, y sólo para la relación entre renta y exportaciones ${ }^{8}$. España está prácticamente ausente de estos estudios, sólo se obtienen resultados en Ghali, y es para los años más recientes ${ }^{9}$.

Para evitar problemas por omisión de variables, el análisis de causalidad entre comercio exterior y crecimiento debería hacerse en el marco más amplio del análisis de los determinantes del crecimiento económico. Así, numerosos autores incluyen variables de comercio exterior o de apertura externa además de las que representan los principales factores de crecimiento, capital físico y capital humano. No obstante, dentro de esta corriente se plantea a menudo la alternativa de analizar de forma aislada las relaciones entre flujos comerciales y renta. Aunque los resultados así obtenidos puedan enmascarar vínculos más complejos entre las variables, ésta es la opción elegida en el presente trabajo. La razón principal radica en que una de sus intenciones es establecer relaciones para una larga etapa que parte del último tercio del siglo $\mathrm{xIx}$, foco de varias controversias sobre el papel del sector exterior en el desarrollo español. Para hacer comparables las estimaciones a lo largo del tiempo, se manejan las mismas variables en todos los períodos analizados, y la escasez de datos para introducir el resto de las variables que determinan el crecimiento en las primeras etapas examinadas obliga a restringir la investigación a la relación entre crecimiento económico y comercio exterior, considerándola como la fase

${ }^{7}$ Giles et al. (1992); Bahmani-Oskooee y Alse (1993); Liu, Song y Romilly (1997) o Asfu-Adjaye y Chakraborty (1999), entre otros. Modelos más completos aparecen en Sharma y Dhakal (1994) o Ghali (1998).

"Serletis (1992), Afxentiou y Serletis (1992), Oxley (1993), Henriques y Sadorsky (1996) y Thornton (1997).

9 Ghali (1998) emplea datos trimestrales entre 1970 y 1994, con la renta y las tasas de inversión, gasto público, exportaciones e importaciones sobre el PIB. Greenaway y Sapsford (1994) incluyen a España en su análisis de episodios de liberalización, diferente al que aquí se realiza, con el resultado excepcional, dentro de su muestra, de un efecto positivo en la constante y negativo en la tendencia de la liberalización de principios de los sesenta, que no llegan a explicar. 
preliminar para un estudio más amplio cuando se consigan mejores indicadores. Esta omisión de variables hace especialmente necesarias las precauciones en la interpretación de los resultados. En el siguiente apartado se detalla el método aplicado para el análisis de tales relaciones.

En cuanto al debate sobre la contribución del comercio exterior al crecimiento económico español, se podría esquematizar siguiendo la división temporal utilizada en el resto del trabajo. En el análisis de lo sucedido entre mediados del siglo XIX y el primer tercio del $\mathrm{xx}$, se ha pasado, de una visión negativa de los efectos de la inserción española en el comercio internacional desde una posición de atraso que determinaba su especialización en productos primarios, como la que presentan Sánchez Albornoz o Nadal, a otra en la que se insiste en las externalidades positivas de las exportaciones españolas, si bien matizadas por el pequeño tamaño relativo del sector exportador que limitaba los efectos de arrastre sobre el conjunto de la economía, como se recoge en los trabajos de Prados de la Escosura y Tortella ${ }^{10}$. La postura de varios autores - como Prados de la Escosura- es que una mayor apertura comercial hubiera ayudado más al crecimiento español ${ }^{11}$. Posteriormente, las políticas de cierre al exterior emprendidas desde 1939 llevaron a una serie de estrangulamientos comerciales y productivos que han sido reiteradamente comentados, especialmente por Donges ${ }^{12}$. En términos globales, la pérdida de peso del comercio exterior en la economía apuntaría así a efectos de baja intensidad sobre el crecimiento. Sin embargo, una característica de esta primera etapa fue la importancia de las exportaciones como fuente de divisas fundamental a la hora de conseguir financiar las tan necesarias importaciones de todo tipo (productos básicos, materias primas y bienes de equipo); de ahí su potencial contribución al desarrollo ${ }^{13}$. Este panorama cambió con la progresiva liberalización y las transformaciones estructurales que tuvieron lugar desde la década de los sesenta, permitiendo un aumento espectacular en los intercambios exteriores y un cambio importante en la especialización comercial. Se ha destacado la importancia de esta apertura para relajar parte de las restricciones de divisas, permitir una mayor importación de

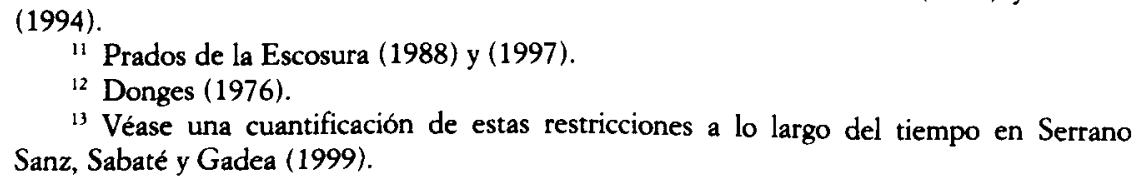
(1994).

11 Prados de la Escosura (1988) y (1997).

12 Donges (1976).

${ }^{13}$ Véase una cuantificación de estas restricciones a lo largo del tiempo en Serrano Sanz, Sabaté y Gadea (1999).

${ }^{10}$ Sánchez-Albornoz (1968), Nadal (1975), Prados de la Escosura (1988) y Tortella 
productos fundamentales para la industria española, y como fuente de una importante difusión de tecnología en los últimos años ${ }^{14}$.

Teniendo en cuenta estas ideas, la influencia del comercio exterior en el crecimiento español debería ser más clara en las últimas décadas, por el elevado y sostenido nivel de apertura alcanzado y porque los vínculos a través de la incorporación tecnológica y sus externalidades son más obvios que en épocas pasadas, tanto en exportaciones como en importaciones. En el resto del artículo se intenta comprobar si los resultados confirman esta visión, y en qué dirección de causalidad se plasmaba el previsiblemente menor potencial de influencia de los flujos comerciales en las etapas anteriores.

\section{RESULTADOS DEL ANÁLISIS DE CAUSALIDAD}

De acuerdo con las hipótesis que se han citado anteriormente, se emplean a continuación técnicas de cointegración y representación en forma de mecanismo de corrección del error (MCE) para aplicar los contrastes de causalidad tipo Granger a la relación entre renta y exportaciones, por un lado, y entre renta e importaciones, por otro. Como es conocido, el test de causalidad de Granger contrasta si los cambios en una variable preceden a los cambios en otra. Así, una variable $\mathrm{X}$ causa a otra $\mathrm{Y}$ en el sentido de Granger si se predice mejor la evolución de $\mathrm{Y}$ teniendo en cuenta la información pasada de $\mathrm{X}$ que sin tenerla en consideración. Pero cuando las variables no son estacionarias (como sucede a menudo con las series económicas) y están cointegradas, los tests de Granger habituales no son válidos, y es necesario incluir en ellos la información sobre el ajuste respecto a la relación de largo plazo que se establece entre las variables ${ }^{15}$. Por lo tanto, la comprobación de dicha causalidad deberá basarse en un análisis previo de las propiedades temporales de las series empleadas y de las posibles relaciones de equilibrio a largo plazo entre ellas.

${ }^{14}$ Tortella (1994) y Alonso y Donoso (1999).

15 Granger (1988). Es decir, entre variables estacionarias se aplica un test de tipo F para comprobar la significatividad de una variable en la explicación de la dependiente. Entre variables no estacionarias el test se establece con dichas variables en diferencias. Para variables integradas de orden uno cointegradas entre sí, se incorpora la desviación respecto a la relación de equilibrio a largo plazo que las une (el término denominado de corrección del error), como se observa en las siguientes expresiones, que recogen la relación entre renta y exportaciones (renta e importaciones se relacionan de forma análoga): 
La ausencia de series españolas de comercio exterior ininterrumpidas impide una estimación única desde el último tercio del siglo XIX hasta finales del siglo $\mathrm{xx}$, que permitiría analizar relaciones de largo plazo estrictamente hablando y comparaciones con ciclos más breves. Teniendo en cuenta esta consideración, junto con los períodos habituales para el análisis del sector exterior español, se divide la aplicación del método de trabajo recién expuesto en tres etapas. Corresponde la primera al último tercio del siglo XIX y el primero del siglo XX, de 1870 a 1935; la segunda, a la etapa autárquica, de 1940 a 1959, y la tercera, a los años del desarrollo económico y la integración en Europa, que por los datos empleados se limitan a la etapa 1964-1995. En el Apéndice (pp. 166-171), se detallan las series utilizadas y el proceso de estimación en cada período, de forma que, a continuación, en el Cuadro 1 , se resumen los resultados más destacados.

En la etapa previa a la Guerra Civil, la renta causaba las exportaciones en el sentido de Granger y no al contrario, y además también influía en las importaciones. Esto contradice las hipótesis más optimistas sobre el influjo del comercio exterior sobre el crecimiento económico español, pero resulta coherente con las características de nuestros intercambios exteriores en términos globales. A pesar de los cambios ocurridos en varios sectores, se podría caracterizar al comercio de esta época por su elevada variabilidad y vulnerabilidad ante cambios externos - climáticos o tecnológicos, entre otros- comparado con épocas más recientes, lo que restaría influencia sobre el crecimiento interno. Adicionalmente, la composición del sector exportador español (alimentos sin elaborar y minerales, principalmente) no era la más adecuada para que su desarrollo conllevara aumentos significativos de productividad a través de una mejor y más rápida difusión de la tecnología exterior, ni tampoco para aprovechar la explotación de

$$
\begin{aligned}
& \Delta Y_{t}=a_{0}+\sum_{i=1}^{n} \alpha_{i} \Delta Y_{t-i}+\sum_{i=1}^{n} \gamma_{i} \Delta X_{t-i}+\delta M C E_{t-i}+\varepsilon_{t} \\
& \Delta X_{t}=b_{0}+\sum_{i=1}^{n} \beta_{i} \Delta X_{t-i}+\sum_{i=1}^{n} \sigma_{i} \Delta Y_{t-i}+\theta M C E_{t-1}+\mu_{t}
\end{aligned}
$$

En la primera ecuación $\mathrm{X}$ causa-Granger $\mathrm{Y}$ si algún coeficiente de $\Delta \mathrm{X}$ es distinto de cero, pero también si $\delta$ es significativo. Las variables se toman en logaritmos, los términos de error son ruido blanco, $\Delta$ representa el operador diferencias y MCE es el término de corrección del error. Los estadísticos $F$ se calculan bajo la hipótesis nula de que en la primera (segunda) ecuación todos los $\gamma(\sigma)$ son igual a cero. 
CUADRO 1

Test de causalidad de Granger con MCE (estimación MCO)

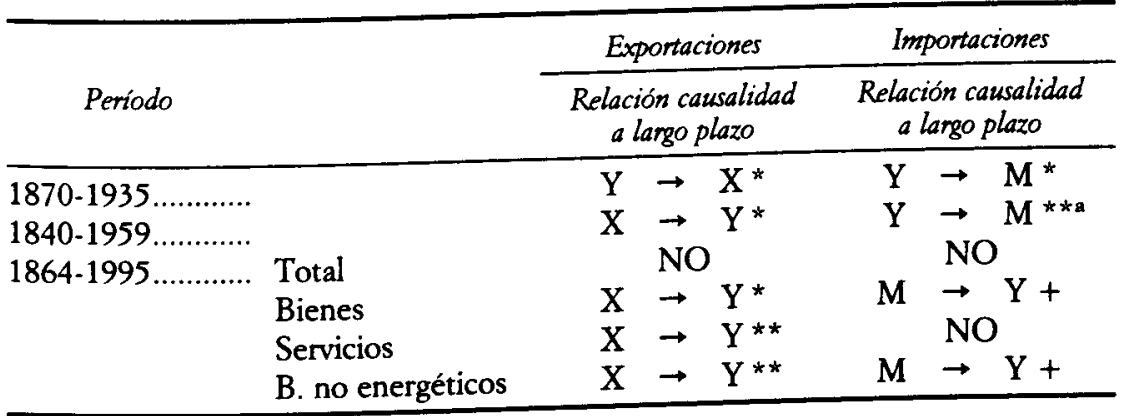

NOtAs: a Aparece la misma relación en el corto plazo. Niveles de significación: +10 por $100 ;{ }^{*} 5$ por $100 ;{ }^{* *} 1$ por 100.

FUENTE: Véase el texto.

economías de escala que podían estar ya presentes en algunas ramas manufactureras ${ }^{16}$. Estos rasgos podrían explicar que, a pesar de su diferente nivel de desarrollo y apertura, en el único trabajo comparable encontrado, para la etapa 1850-1913, se encuentre la misma relación entre exportaciones y renta estimada en el caso de Gran Bretaña, mientras que la relación resulta bidireccional para Dinamarca y Alemania, y son las exportaciones las que causan la renta en sentido Granger en Italia, Noruega y Suecia ${ }^{17}$.

En el período autárquico, los resultados obtenidos concuerdan con algu. nas características conocidas de esta etapa: de nuevo, el lado de la demanda explica la relación entre renta e importaciones, pero en este caso está presente la causalidad esperada de las exportaciones hacia la renta. No obstante, dados los escasos cambios en la especialización comercial española hasta 1959, esta dirección de causalidad vendría más bien explicada en

${ }^{16}$ Tortella (1994), por ejemplo, insiste en la baja contribución de las exportaciones agrícolas a las primeras etapas de crecimiento español. Por otra parte, varios autores han identificado el tipo de efectos positivos mencionados para sectores exportadores concretos, como la minería (Prados de la Escosura, 1982) o las naranjas (Simpson, 1992), pero han hecho notar su escaso alcance para el resto de la economía.

17 Thornton (1997). Pero debe tenerse en cuenta que en las numerosas estimaciones para países desarrollados y en desarrollo en etapas más recientes tampoco se encuentra un patrón de causalidad claro ligado a las diferencias en los niveles de renta o de apertura. Véase Sharma y Dhakal (1994). 
términos de suavización de la restricción de divisas a través de las ventas al exterior, lo que permitiría a su vez mayores importaciones ${ }^{18}$.

Desde los años sesenta, las estadísticas permiten apreciar las relaciones entre varios tipos de flujos comerciales y la renta. La causalidad tipo Granger va de las exportaciones de servicios a la renta. Dado el predominio del sector turístico en nuestras exportaciones, la interpretación de dicha causalidad es de nuevo en términos de consecución de divisas más que de incrementos de productividad. Pero en esta etapa también aparece en el comercio de mercancías la dirección de causalidad que se esperaba, del comercio hacia la renta, con mayor rotundidad en las exportaciones que en las importaciones, y tanto incluyendo el comercio de productos energéticos como eliminándolo. A pesar de las diferencias en las variables y período utilizados, estos resultados coinciden con los conseguidos en el único trabajo que nos puede servir de referencia para el caso español, donde se obtiene que exportaciones e importaciones causan la renta (aunque también causalidad de la renta a las importaciones) ${ }^{19}$. Esta causalidad desde ambos tipos de flujos al crecimiento aparece también en otros países desarrollados: Francia, Japón, Suiza y Reino Unido, mientras que Italia sólo comparte la causalidad de exportaciones a renta, y Estados Unidos, Australia y Canadá desde las importaciones a la renta ${ }^{20}$. De esta forma, a través de un comercio que desde hace años se compone ya fundamentalmente de manufacturas que se intercambian en su mayor parte con economías desarrolladas, podría afirmarse, de acuerdo con la bibliografía revi-

${ }^{18}$ Los efectos de la escasez de divisas como freno al crecimiento de la economía española en esta etapa se cuantifican en Serrano Sanz (1997) y Serrano Sanz, Sabaté y Gadea (1999). Sin embargo, el análisis de causalidad entre exportaciones e importaciones con los datos aqui manejados da como resultado que las importaciones influian en las exportaciones (incluyendo una variable ficticia desde 1956), mientras sucede lo contrario para los otros dos períodos de estudio. Esto obliga a desconfiar de los escasos datos utilizados para la autarquía, y a plantear para el futuro estimaciones multivariantes que permitan detectar relaciones indirectas entre las variables $u$ otros posibles determinantes. Estos resultados no se han incluido por razones de espacio.

${ }^{19}$ Ghali (1998).

20 En Francia, Suiza, Estados Unidos, Canadá y Noruega aparece también la influencia de la renta en las exportaciones. Con nuestros datos no se observa esta causalidad de doble dirección entre comercio y renta, aunque no ha sido rara en estudios sobre países en desarrollo en las últimas tres décadas. Éste es el caso en Bahmani-Oskooee y Alse (1993), para ocho países en desarrollo que incluyen a Grecia; o en Sharma y Dhakal (1994), con treinta países incluyendo a Grecia (para el que la causalidad actúa de las exportaciones a la renta) y Portugal (con causalidad de doble dirección entre renta y exportaciones). En este último trabajo hay cinco países con esta característica, y otros tantos que muestran cada una de las dos direcciones por separado. De nuevo hay que insistir en la falta de un patrón claro que se relacione con el grado de desarrollo. 
sada, que se consigue un aumento de la difusión tecnológica y, con ella, de la productividad de la economía española.

Resulta muy difícil encontrar similitudes claras entre nuestros resultados y los observados para países de nuestro entorno a lo largo del tiempo, por las diferencias en especificaciones y períodos, y por la falta de un patrón empírico claro a nivel internacional ${ }^{21}$. Con muchas precauciones, por lo tanto, podríamos señalar cierto parecido con los casos portugués y británico, puesto que los resultados más recientes apuntan a la influencia de las exportaciones en su crecimiento, mientras que remontándose al siglo XIX se obtenía la relación contraria, pero poco más puede aventurarse en esta dirección con los datos disponibles hasta ahora ${ }^{22}$.

\section{CONCLUSIONES}

A pesar de las diferencias señaladas, un resultado consistente en todos los períodos ha sido el signo positivo en todas las relaciones entre comercio y renta que se han estimado. Es decir, que a lo largo del último siglo ha predominado una influencia positiva en el crecimiento económico, rechazándose así las hipótesis del perjuicio causado a la economía española por el comercio exterior. Por otro lado, y a diferencia de algunos trabajos, el comportamiento de exportaciones e importaciones no es idéntico, lo que permite negar que representen sin más el comercio exterior, sino que ambos tienen una relación separada con la evolución de la renta ${ }^{23}$. Siguiendo el esquema temporal que ha servido de hilo conductor, se concluye que la influencia entre flujos de comercio y crecimiento económico en la economía española no ha permanecido inalterada, lo que concuerda con la evolución de su sector exterior tanto en peso relativo como en

${ }^{21}$ Encontramos dos trabajos que abarcan conjuntamente toda la etapa considerada en este análisis. En Portugal, para el período 1865-1985 se encuentra una relación de causalidad de la renta a las exportaciones (la contraria sólo al 10 por 100 de significación). Oxley (1993). Para Canadá se han realizado varias estimaciones entre 1870 y 1990 , en las que predomina la causalidad de exportaciones a renta (y a importaciones) pero no desde la postguerra en Serletis (1992) y tampoco desde finales del xIX en Afxentiou y Serletis (1992); estos últimos resultados coinciden con los de Ghali (1998) para las últimas décadas. Henriques y Sadorsky (1996) obtienen que la renta causa las exportaciones, pero su test de Granger es incorrecto porque no incluye el mecanismo de corrección del error.

${ }^{22}$ Con los datos mencionados de Oxley (1993) y Sharma y Dhakal (1994) para Portugal, y Thornton (1997) junto a Ghali (1998), para el Reino Unido. (1992). 
su composición a lo largo del tiempo. Estos cambios ayudan a explicar el incumplimiento de las hipótesis generales planteadas inicialmente en alguna de las etapas que se analizan ${ }^{24}$.

En primer lugar, la causalidad que se observa desde finales del Diecinueve hasta la década de los cincuenta, de la renta a las importaciones, es un resultado plausible. La conocida rigidez de nuestras compras externas de todo tipo, ante el relativo atraso de nuestra economía, hace que durante este largo período predomine el tirón de la demanda como motor de las importaciones, y no'se observe un efecto determinante de éstas sobre la renta. Recordemos que en su composición se incluían productos con mayor contenido tecnológico que los nacionales, pero también que hasta finales de los años cincuenta predominaron las compras de materias primas y alimentos.

La relación causal de la renta hacia las exportaciones aparece únicamente para la etapa previa a la Guerra Civil. Aunque podría haberse esperado también el efecto contrario, de acuerdo con parte de las teorías aludidas en el primer epígrafe, de modo que el aprovechamiento de la demanda externa y las ventajas dinámicas a ella ligadas impulsasen el crecimiento, estas relaciones no se plasmaron así en la práctica para la economía española del XIx. El resultado obtenido concuerda con un papel como máximo modesto de las externalidades de las exportaciones, no incompatible con los efectos positivos en sectores concretos, pero que no llega a reflejarse en un efecto de arrastre para el conjunto de la economía. Esta relación de causalidad puede estar evidenciando, al menos en parte, la utilización de las exportaciones como salida del excedente, respondiendo al tirón de la demanda externa en algunos períodos. En cualquier caso, estos resultados no sirven para zanjar la discusión sobre el papel potencial del sector exterior en el crecimiento español. Lo que sugieren es que, con las exportaciones que se dieron en la realidad (es decir, la especialización existente, el peso relativo de los sectores exportadores dentro de la economía nacional y las deficiencias de integración del mercado interno) no se apoyaría una estrategia para impulsar el crecimiento económico basado en las exportaciones. Autores como Federico (1992), sin defender la tesis de dependencia (que nuestros resultados rechazan), afirman que los países mediterráneos poseían escasa capacidad para aprovechar los estímulos de la

${ }^{24}$ Para comprobar la robustez de los resultados, se han repetido las estimaciones considerando tasas de exportaciones y de importaciones sobre PIB, y renta per cápita, para todos los períodos. En esencia coinciden con lo presentado, si bien aparece algún caso de causalidad bidireccional. 
demanda de los países más adelantados, y que las bajas elasticidades-renta en sus funciones de exportación alejaban las posibilidades de un crecimiento liderado por las exportaciones ${ }^{25}$. En el caso español esta tendencia puede resultar más clara, dada la mayor elasticidad-renta de las importaciones: las características estructurales implicaban una restricción del sector exterior al crecimiento económico español ${ }^{26}$. Este tipo de consideraciones apuntan hacia un modesto papel del comercio exterior en el crecimiento de este período.

La dirección de causalidad cambia para las exportaciones ya durante el período autárquico, probablemente indicando la importancia de las escasas exportaciones para relajar la «sed de divisas» que estrangulaba las posibilidades de crecimiento en la etapa. A partir de los años sesenta las exportaciones de servicios son parte fundamental en ese alivio, lo que corrobora de nuevo la relación causal. Pero también se refleja en las exportaciones de mercancías. Dado el cambio producido en la especialización española en las últimas décadas (y la mayor amplitud de recursos de la balanza de pagos para cubrir déficit comerciales), ahora sí podría aceptarse una interpretación en términos de efectos dinámicos sobre el crecimiento, tanto por las economías de escala (España se ha especializado en sectores intensivos en las mismas) como por la difusión de conocimientos, que también se originaría por el lado de las importaciones, aunque los resultados son menos firmes en este caso.

Estas conclusiones están sujetas a varias matizaciones. No debe olvidarse que el ejercicio llevado a cabo sólo identifica relaciones de causalidad de tipo Granger entre dos variables, pero no pretende que crecimiento y comercio exterior sean la única explicación uno del otro. Una de las revisiones que debe plantearse consiste en solucionar el problema de las variables omitidas en la relación, considerando modelos de crecimiento más completos. Esta vía es, por supuesto, más factible para etapas recientes, por la mayor disponibilidad de datos sobre dichos determinantes del crecimiento. Una forma de completar el análisis que podría aplicarse a todo el período sería incluir el nivel de apertura o liberalización comercial de

${ }^{25}$ Federico (1992). La significatividad de la renta externa como determinante principal de las exportaciones se observa en Prados de la Escosura (1988) para el período 1821-1913; en Herranz y Tirado (1996) para 1870-1913, y en Serrano Sanz et al. (2001), para 1870-1935. En estos dos últimos casos se recogen las mencionadas bajas elasticidades. Puede recordarse, además, que la excepcional situación de las exportaciones españolas en la década de 1880 no pudo mantenerse más allá de final de siglo. No obstante, autores como Fraile (1992) y Prados de la Escosura (1997) defienden conclusiones contrarias.

${ }^{26}$ Como se confirma en Herranz y Tirado (1996) y Serrano Sanz (1997). 
la economía española, y comprobar su relación con sus etapas de crecimiento y de cambio estructural. Una segunda vía, también para todo el período considerado, sería distinguir la influencia en el crecimiento de la composición de exportaciones e importaciones en las distintas etapas, puesto que se hace depender de la especialización comercial española y de sus cambios, la explicación de algunas de las relaciones obtenidas hasta ahora.

\section{APÉNDICE \\ Estimación de los tests de causalidad de Granger}

En primer lugar, debe comprobarse el orden de integración de las variables. El análisis de raíces unitarias muestra que casi todas las series son integradas de orden uno, aunque a veces sólo al 10 por 100 . Sin embargo, para el primer período, exportaciones e importaciones resultan estacionarias, mientras la renta parece integrada de orden uno. Para poder establecer una relación entre las variables, se comprueba si la renta es, en realidad, una variable estacionaria con algún tipo de ruptura estructural. La aplicación de los test propuestos por Vogelsang y Perron (1994) y Perron (1994) para buscar cortes estructurales no determinados exógenamente permite descubrir la posibilidad de dicho corte, con un cambio en tendencia a partir de 1918, aunque sólo al 10 por 100 de significación, y, por lo tanto, aceptar que se trata de una serie $\mathrm{I}(0)^{27}$. En segundo lugar, se realiza la búsqueda de relaciones de cointegración entre las variables integradas de orden uno, a través de los tests multivariantes tipo Johansen (1988) ${ }^{28}$. Si no se detecta ningún vector de cointegración, se pueden aplicar los tests de Granger tradicionales para analizar la causalidad entre las variables. Si se detecta un vector, ello garantiza que existe causalidad al menos en una dirección (por el teorema de representación de Granger). Se aplica el tests de Granger reintroduciendo la información del largo plazo (mediante el término MCE) en el análisis de corto plazo (como en la nota 15) ${ }^{29}$. Ello permite establecer la dirección de dicha causalidad, que se observa

${ }^{27}$ Valor del estadístico: -4,59; valor crítico al 10 por 100 en Perron (1994): $-4,48$ (modelo de ruptura en tendencia); año de ruptura: 1917.

${ }^{28}$ El número de retardos para el sistema VAR inicial combina los criterios de información de Akaike y Schwarz-Bayes con la necesidad de ausencia de correlación en las ecuaciones.

${ }^{29}$ Los tests de tipo Granger son sensibles a la variación en los retardos de las ecuaciones que se utilizan. Serletis (1992). El método que aquí se sigue para elegir su número óptimo 
de dos formas: 1) si el término MCE es significativo, lo que muestra que las variables independientes causan la dependiente. En este caso se estaría contrastando la causalidad a largo plazo entre las variables; y 2) aplicando los test de Granger a los retardos de las variables en diferencias para comprobar si son significativos. De este modo se contrasta la causalidad en el corto plazo ${ }^{30}$.

Para la etapa 1870-1935, junto a los datos de renta nacional de Prados de la Escosura (1995), se han utilizado como series de exportaciones e importaciones reales los índices de volumen de Prados de la Escosura (1986) para los años 1870-1900, enlazados con los del INE (1958) a partir de esa fecha. Como se ha mencionado, el test de Granger entre variables que resultan estacionarias se limita al caso más sencillo, aunque teniendo en cuenta la variable ficticia que recoge la ruptura en la serie de renta a partir del final de la Primera Guerra Mundial. Los resultados indican relaciones de causalidad que van desde la renta a las exportaciones y a las importaciones; en este último caso se hace necesario introducir una variable ficticia que recoge la importante caída de las importaciones durante la Gran Guerra, en concreto para los años 1916-1918 (Cuadros 3 y 4) ${ }^{31}$.

Los datos utilizados para el período de la autarquía, 1940-1959, proceden de Prados de la Escosura (1995) en el caso de la renta, y del INE (1958) enlazado con los Anuarios Estadísticos del INE desde 1956 para las series de comercio real. En las relaciones entre exportaciones y renta se identifica un vector de cointegración sin grandes problemas, al incluir una variable ficticia para recoger la súbita caída de las ventas exteriores en 1956 (atribuible, fundamentalmente, a problemas en la cosecha de naranjas). La aplicación de los tests de Granger con la información contenida en la relación de largo plazo da como resultado que las exportaciones causaban la renta en el largo plazo (Cuadros 2 y 3). En las importaciones,

es minimizar el error final de predicción a través del estadístico de Akaike, de forma sucesiva para la variable explicada y la independiente. Véase Sharma y Dhakal (1994) como ejemplo.

${ }^{30}$ Debe mencionarse que algunos de los problemas que surgen para detectar las relaciones de causalidad pueden atribuirse a que se trabaja con pocas observaciones - lo que en nuestro caso podría afectar al período final y, sobre todo, a la fase de autarquía - o al uso de datos anuales en lugar de una periodicidad inferior. En varias ocasiones ha sido necesario recurrir al uso de variables ficticias para detectar la causalidad con claridad, como se señala en el resto del Apéndice.

${ }^{31}$ Las estimaciones se han repetido para el período más reducido 1870-1913, obteniéndose los mismos resultados, tanto con las series utilizadas hasta ahora como con los datos de comercio real de Prados de la Escosura (1982) hasta 1913. En esta etapa más breve, las series resultan integradas de orden uno, y para obtener una relación de coin. tegración entre ellas es necesario recurrir de nuevo a variables ficticias. 


\section{CUADRO 2}

Número de vectores de cointegración y nivel de significación

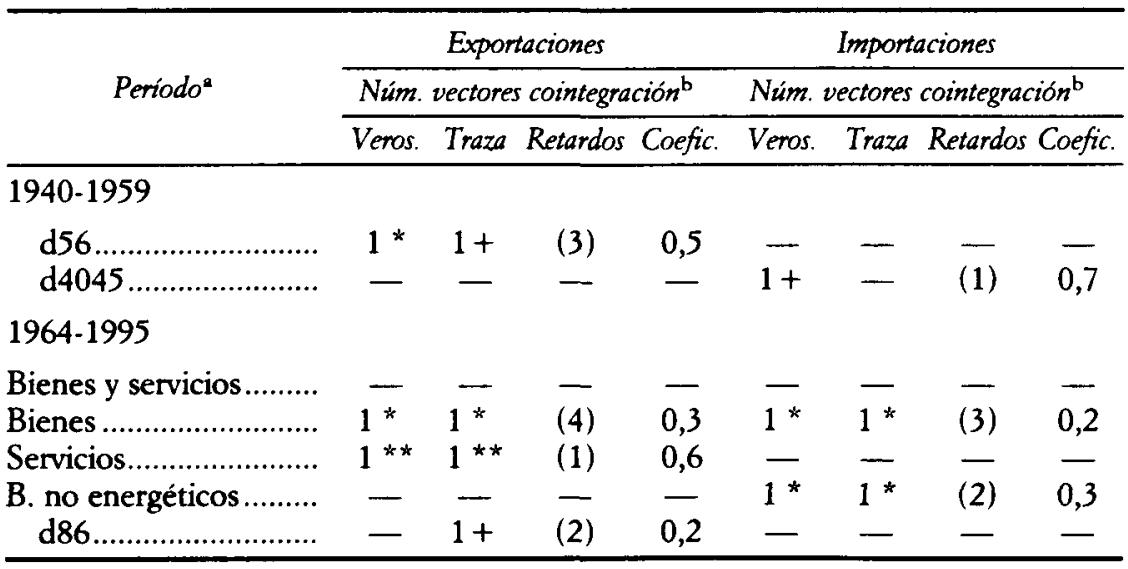

Notas: "Se incluyen las siguientes variables ficticias de tipo escalón en algunos casos: d56 (1956-1959); d4045 (1940-1945); d86 (1986-1995).

bNúmero de vectores de cointegración a partir de los tests de verosimilitud y de traza, respectivamente.

Niveles de significación: +10 por $100 ;{ }^{*} 5$ por $100 ;{ }^{*} 1$ por 100 .

FUENTE: Véase el texto de este Apéndice.

las principales oscilaciones coinciden con los años iniciales del período, en los que la renta española iniciaba su recuperación mientras las importaciones caían hasta el final de la Segunda Guerra Mundial. Si se introduce una variable ficticia que identifique dicho período (1940-1945), podemos analizar la causalidad gracias a la existencia de un vector de cointegración (aunque sólo al 10 por 100 de significación). El resultado es una dirección de causalidad desde la renta a las importaciones, en este caso a largo y corto plazo (Cuadros 2 y 4 ).

Para la estimación de la etapa 1964-1995, se utilizan las series de renta y flujos comerciales en pesetas constantes homogeneizadas por el INE (base 1986) desde 1971, y reconstruidas para 1960-1997 de acuerdo con los índices de crecimiento de la Contabilidad Nacional. Se dispone de series de intercambios exteriores tanto de bienes y servicios conjuntamente como por separado. De forma adicional, se puede recurrir a series de mercancías eliminando el comercio energético, aunque la contrapartida al uso de estas series es que sólo están disponibles para los años 1964-1995, y se eliminan 


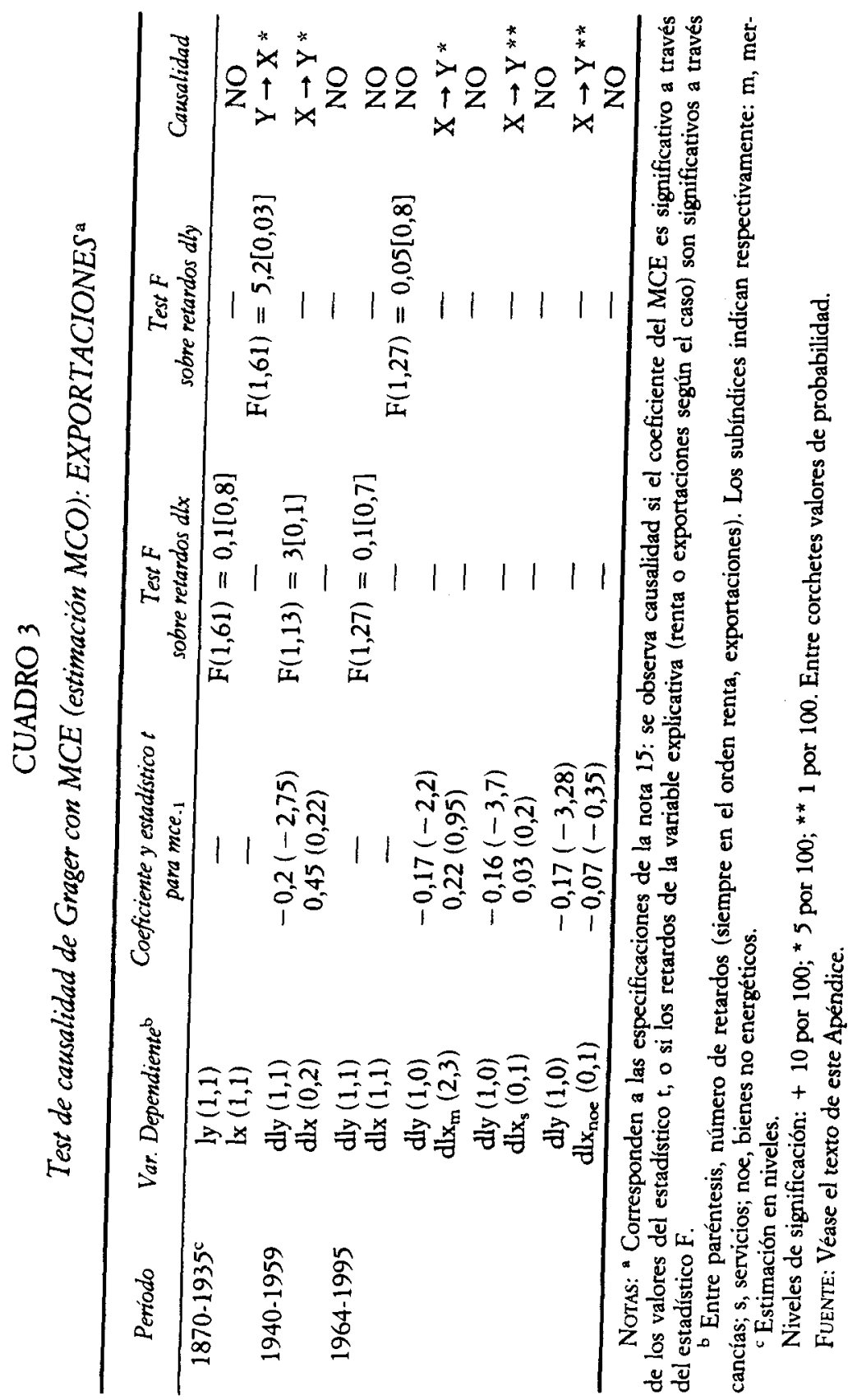




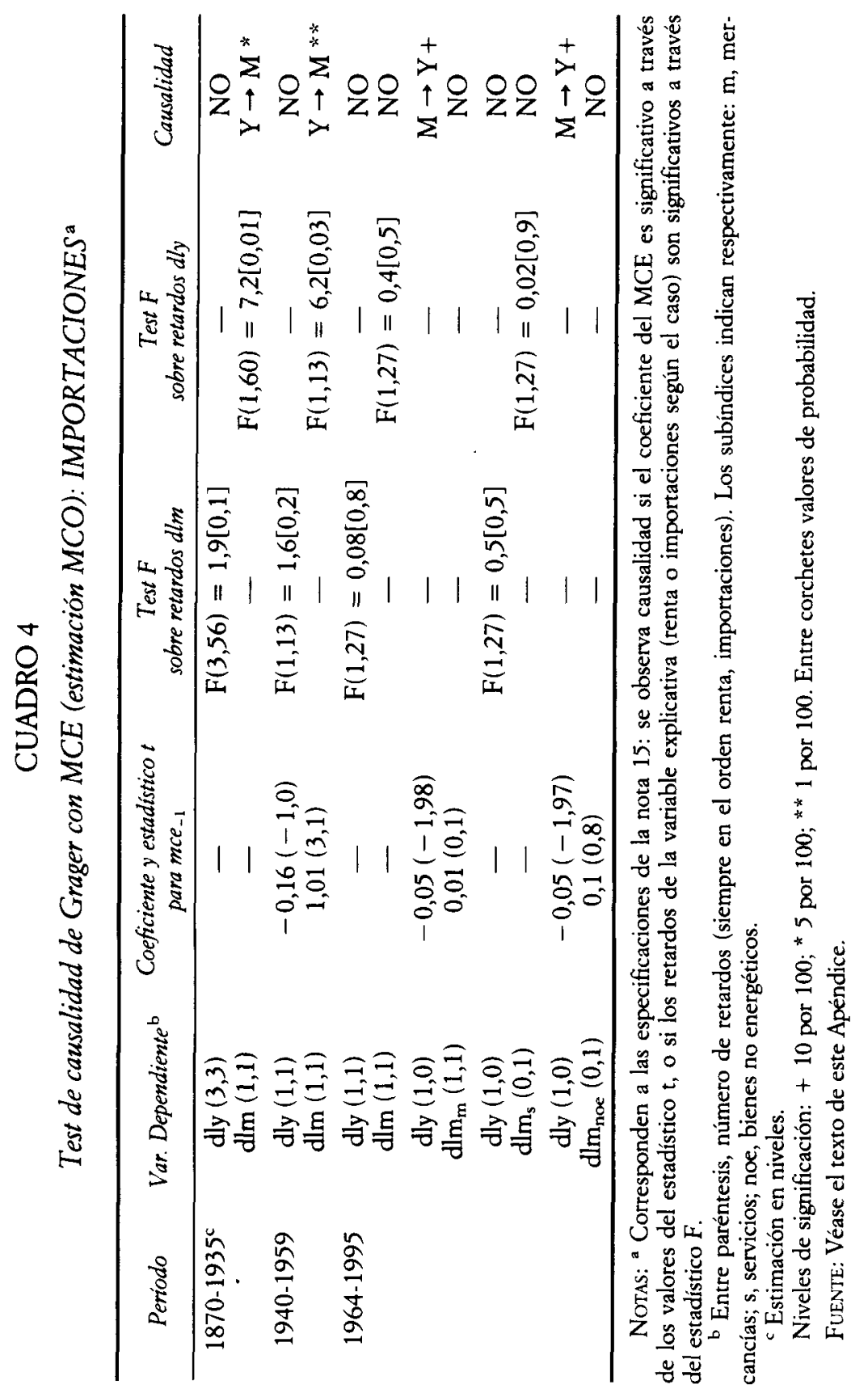


así los primeros años sesenta y 1996-1997 que sí estarían cubiertos por el resto de las series Para explotar todos los datos disponibles con un tratamiento temporal homogéneo, se han realizado todas las estimaciones en la etapa 1964-1995.

No se identifica una relación de largo plazo entre renta y flujos de comercio total en el período. En consecuencia, deben aplicarse los tests de causalidad de Granger tradicionales, en términos de diferencias en las variables. Su resultado es la ausencia de causalidad en ninguna dirección. Pero estas series no son equiparables a las que aparecían para los períodos previos, puesto que incluyen datos sobre servicios que, como es conocido, fueron adquiriendo un peso creciente en nuestros flujos exteriores. Por esta razón se analizan las relaciones de causalidad de mercancías y de servicios por separado. A diferencia de la estimación referente al total de bienes y servicios, aparece una relación clara de cointegración entre renta y exportaciones de mercancías y otra entre renta e importaciones (Cuadro 2). La dirección de causalidad que se observa es desde las exportaciones a la renta. Las importaciones también causan la renta en sentido Granger, pero sólo al 10 por 100 de significación (Cuadros 3 y 4). Cuando se repite el análisis para los flujos de servicios, la relación de largo plazo entre exportaciones y renta y la dirección de causalidad son claras, en esta ocasión fluye de las exportaciones de servicios hacia la renta. Por el contrario, no se confirma ni la existencia de una relación de equilibrio ni de causalidad entre compras de servicios al exterior y renta nacional (Cuadros 2 y 4). Por último, la posibilidad de distorsiones en los datos debido a la rigidez en el comercio de productos energéticos, conduce a eliminarlos de la regresión. En este caso es más difícil identificar la relación de largo plazo para las exportaciones (un vector de cointegración sólo al 10 por 100 de significación cuando se incluye una ficticia para el período posterior a 1986) que para las importaciones (al 5 por 100$)^{32}$. Pero ahora, del análisis de causalidad se deduce que la dirección va siempre del comercio hacia la renta, y no viceversa, si bien la relación es clara en el caso de las exportaciones y sólo marginal (al 10 por 100) para las importaciones (Cua. dros 3 y 4).

32 Un resumen de la discusión sobre la existencia o no de cambio estructural a partir de 1986 en las funciones de comercio exterior se presenta en Serrano Sanz et al. (2001). 


\section{BIBLIOGRAFÍA}

AfXentiou, P. C., y Serletis, A. (1992): «Openness in the Canadian economy, 1870-1988», Applied Economics, 24, 11, pp. 1191-1198.

Alonso, J. A., y Donoso, V. (1999): «Sector exterior: apertura económica y líneas de investigación», en J. L. García Delgado (dir.), España, economia: ante el siglo XXI, Madrid, Espasa Calpe, pp. 207-239.

ASFU-ADJAYE, J., y ChAKRABORTY, D. (1999): «Export-Led Growth and Import Compression: Further Time Series Evidence from LDCs», Australian Economic Papers, 38, 2, pp. 164-175.

Bahmani-OskoOeE, M., y Alse, J. (1993): «Export Growth and Economic Growth: An Application of Cointegration and Error-Correction Modeling», The Journal of Developing Areas, 27, 4, pp. 535-542.

Barro, R., y SAlA-I-Martin, V. (1995): Economic Growth, New York, McGraw-Hill.

Bhagwati, J. (1988): Protectionism, Cambridge, MIT Press.

Bhagwati, J., y Srinivasan, T. (1979): «Trade Policy and Development», en R. DORnBuSCH y J. Frenkel (eds.), Intermational Economic Policy: Theory and evidence, Baltimore, John Hopkins Press, pp. 1-35.

DONGES, J. (1976): La industrialización en España, Barcelona, Oikos-Tau.

EDWARDS, S. (1993): «Openness, Trade Liberalization, and Growth in Developing Countries», Joumal of Economic Literature, 31, pp. 1358-1393.

FEDERICO, G. (1992): «El comercio exterior de los países mediterráneos en el siglo XXX», en L. PRADOS DE LA Escosura y V. ZAMAGNi (eds.), El desarrollo económico en la Europa del Sur: España e Italia en perspectiva histórica, Madrid, Alianza, pp. 269-292.

Fraile Balbin, P. (1992): «Los mercados del Centro y las economías atrasadas de Europa, 1900-1930», en L. Prados y V. ZamaGNi (eds.), El desarrollo económico en la Europa del Sur: España e Italia en perspectiva histórica, Madrid, Alianza, pp. 253-266.

GHALI, K. H. (1998): «Government Size and Economic Growth: Evidence from a Multivariate Cointegration Analysis», Applied Economics, 31, 8, pp. 975-987.

Gires, D. E. A.; Giles, J. A., y McCanN, E. (1992): «Causality, Unit Roots and Export-Led Growth: Evidence from New Zeland», The Journal of International Trade and Economic Development, 1, 2, pp. 196-218.

Granger, C. W. J. (1988): «Causality, Cointegration and Control», Joumal of Economic Dynamics and Control, 12, pp. 551-559.

GreENAWAY, D., y SAPSFORD, D. (1994): «What Does Liberalisation Do for Exports and Growth?», Weltwirtschaftliches Archiv, 130, 1, pp. 152-173.

Greenaway, D.; Morgan, W., y WRight, P. (1999): «Exports, Export Composition and Growth», The Joumal of International Trade and Economic Development, 8,1, pp. $41-51$.

Grossman, G. M., y Helpman, E. (1991): Innovation and Growth in the Global Economy, Cambridge, MIT Press.

Helpman, E., y Krugman, P. (1985): Market Structure and Foreign Trade, Cambridge, MIT Press. 
Henriques, I., y SADORSky, P. (1996): «Export-Led Growth or Growth-Driven Exports? The Canadian Case», Canadian Joumal of Economics, 29, 3, pp. 540-555.

Herranz, A., y Tirado, D. A. (1996): «La restricción exterior al crecimiento económico español (1870-1913)», Revista de Historia Económica, 14, 1, pp. 11-49.

Instituto Nacional DE Estadística (INE) (1958): Comercio exterior de España. Números indices (1901-1956), Madrid.

- (INE) (varios años), Anuario estadístico de España, Madrid.

JohANSEN, S. (1988): «Statistical Analysis of Cointegration Vectors», Joumal of Economic Dinamics and Control, 12, pp. 231-254.

KRAVIS, I. (1970): «Trade as a Handmaiden of Growth: Similarities Between the Nineteenth and Twentieth Centuries», The Economic Joumal, 80, 320, pp. 850-872.

KRUEGER, A. (1980): «Trade Policy as an Input to Development», American Economic Review, 70, 2, pp. 188-292.

LeVINE, R, y RENELT, D. (1992): «A Sensitivity Analysis of Cross-country Growth Regressions», American Economic Review, 82, 4, pp. 942-963.

LEWIS, W. A. (1978). The Evolution of the International Economic Order, Princeton, Princeton University Press.

LiU, X.; Song, H., y Romilly, P. (1997): «An Empirical Investigation of the Causal Relationship Between Openness and Economic Growth in China», Applied Economics, 29, 12, pp. 1679-1686.

McKinnon, R. (1964): «Foreign Exchange Constraint in Economic Development and Efficient Aid Allocation», Economic Joumal, 74, 294, pp. 388-409.

NaDal, J. (1975): El fracaso de la revolución industrial en España, 18141913, Barcelona, Ariel.

NuRKSE, R. (1964): Equilibrio y crecimiento en la economía mundial, Madrid, Rialp.

OxLEY, L. (1993): «Cointegration, Causality and Export Led Growth in Portugal 1865-1985», Economic Letters, 43, 2, pp. 163-166.

Perron, P. (1994): «Further Evidence on Breaking Trend Functions in Macroeconomic Variables», Working Paper, núm. 2594, CRDE, Université de Montréal.

Prados de la Escosura, L. (1982): Comercio exterior y crecimiento económico en España, 1826-1913: tendencias de largo plazo, Madrid, Banco de España.

- (1986): «Una serie anual del comercio exterior español (1821-1913)», Revista de Historia Económica, 4, 1, pp. 103-150.

- (1988): De Imperio a Nación. Crecimiento y atraso económico en España (1780-1930), Madrid, Alianza.

- (1995): Spain's Gross Domestic Product, 1850-1993: Quantitative conjectures, Documento de Trabajo, Universidad Carlos III, Madrid.

- (1997): «Política económica liberal y crecimiento en la España contemporánea: un argumento contrafactual», Papeles de Economia española, 73, pp. 83-99.

Prados DE la Escosura, L., y ZamaGni, V. (eds.) (1992): El desarrollo económico en la Europa del Sur: España e Italia en perspectiva bistórica, Madrid, Alianza.

Prebisch, R. (1959): The Economic Development of Latin America and its Principal Problem, CEPAL. 
RodriK, D. (1995): «The Political Economy of Trade Policy», en J. Behrman y T. SRINIVASAN (eds.), Handbook of International Economics, vol. 3, Amsterdam, North-Holland, pp. 1457-1496.

SÁNCHEZ-AlboRNOZ, N. (1968): España bace un siglo: una economía dual, Barcelona, Península.

SERLETTS, A. (1992): «Export Growth and Canadian Economic Development», Journal of Development Economics, 38, 1, pp. 133-145.

SERRANo SANZ, J. M. (1997): «Sector exterior y desarrollo en la economía española contemporánea», Papeles de Economia Española, 73, pp. 308-335.

Serrano Sanz, J. M.; SabatE, M., y Gadea, D. (1999): «Economic Growth and the Long Run Balance of Payments Constraint in Spain», Journal of International Trade and Development, 8, 4, pp. 389-417.

Serrano SANZ, J. M.; SABATE, M.; PARDOS, E., et al. (2001): La modernización del sector exterior de la economía española, Proyecto de investigación para la Fundación BBV (manuscrito)

Sharma, S. C, y DhaKal, D. (1994): «Causal Analyses between Exports and Economic Growth in Developing Countries», Applied Economics, 26, 12 , pp. 1145-1157.

SimpSON, J. (1992): «Los límites del crecimiento agrario: España, 1860-1936», en L. Prados de la Escosura y V. Zamagni (eds.), El desarrollo económico en la Europa del Sur: España e Italia en perspectiva bistórica, Madrid, Alianza, pp. 103-138.

SINGER, H. (1950): «The Distribution of Gains between Investing and Borrowing Countries», American Economic Review, 40 (2), pp. 473-485.

ThorNTON, J. (1997): «Exports and Economic Growth: Evidence from 19th Century Europe», Economics Letters, 55, 2, pp. 235-240.

Tortella, G. (1994): El desarrollo de la España Contemporánea. Historia económica de los siglos XIX y XX, Madrid, Alianza.

Vogelsang, T., y Perron, P. (1994): «Additional Tests for Unit Root Allowing for a Break in the Trend Function at an Unknown Time», Dep. of Economics, Cornell University, Ithaca, NY (manuscrito). 